Neema Wilberth, Marco Mpimbo, and Santosh Kumar*

\title{
Somewhere Dense Orbit that is not Dense on a Complex Hilbert Space
}

https://doi.org/10.1515/conop-2019-0005

Received August 2, 2018; accepted April 26, 2019

Abstract: In this paper, we present the existence of $n$-tuple of operators on complex Hilbert space that has a somewhere dense orbit and is not dense. We give the solution to the question stated in [11]: "Is there $n$-tuple of operators on a complex Hilbert space that has a somewhere dense orbit that is not dense?" We do so by extending the results due to Feldman [11] and Leòn-Saavedra [12] to complex Hilbert space. Further illustrative examples of somewhere dense orbits are given to support the results.

Keywords: Orbit, somewhere dense orbit, dense orbit, $n$-tuple, real Hilbert space and complex Hilbert spaces

MSC: 47A16, 47A15.

\section{Introduction}

An $n$-tuple of operators means a finite sequence of length $n$ of commuting continuous linear operators. The concept of orbit comes from the theory of dynamical systems. In the context of operator theory the notion was first used by Rolewicz [14]. Linear dynamics is mainly concerned with the behaviour of iterates of linear transformations. However, a new phenomenon appears in an infinite-dimensional setting: linear operators may have dense orbits. Suppose that $T$ is continuous linear operator on a topological vector space $X$ over the field $\mathbb{F}(=\mathbb{R}$ or $\mathbb{C})$, then for an element $x \in X$, the orbit of $x$ under $T$ is $\operatorname{Orb}(T, x)=\left\{x, T^{1} x, T^{2} x, \ldots\right\}$ where $x \in X$ is a fixed vector. The present work is an extension of the work done by Feldman [11] on Hypercyclicity and somewhere dense orbits.

For more literature on this topic one can see the papers by Al-shami [1], Ansari [2], Ayadi [4], Bayart and Costakis [6], Bayart and Matheron [5], Bourdon and Feldman [7], Costakis and Hadjiloucas [10] and also Costakis and Peris [9].

The concepts of dense orbits is defined as follows:

Definition 1.1. [14] Let $T$ be a continuos linear operator on a topological vectors space $X$. For a fixed element $x \in X$, the orbit of $x$ under $T$ is given by a sequence $T^{n} x: n=0,1, \ldots$.

The following result due to Boyd [8] will be useful in this paper:

Theorem 1.1. [8](Kronecker's theorem) If $x$ is a positive irrational number, then the sequence $\{k x-s: k, s \in \mathbb{N}\}$ is dense in $\mathbb{R}$.

\footnotetext{
Neema Wilberth: Department of Mathematics, College of Natural and Applied Sciences, University of Dar es Salaam, Tanzania, E-mail: neemawilberth@gmail.com

Marco Mpimbo: Department of Mathematics, College of Natural and Applied Sciences, University of Dar es Salaam, Tanzania, E-mail: kmpimbo33@gmail.com

*Corresponding Author: Santosh Kumar: Department of Mathematics, College of Natural and Applied Sciences, University of Dar es Salaam, Tanzania, E-mail: drsengar2002@gmail.com
} 
Remark 1.1. Note that if $x$ is either positive or negative irrational number then $\{k x-s: k, s \in \mathbb{N}\}$ is dense in $\mathbb{R}$.

Below is the theorem due to Feldman [11] that describes the conditions under which somewhere dense orbits are not dense.

Theorem 1.2. ([11]) If $a, b>1$ and $\frac{\ln (a)}{\ln (b)}$ is irrational, then $\left\{\frac{a^{n}}{b^{k}}: n, k \in \mathbb{N}\right\}$ is dense in $\mathbb{R}^{+}$.

The following definitions of Hypercyclicity and multi-hypercyclicity are important as they are part of the discussion in the examples in this section.

Definition 1.2. [3] A continuous linear operator $T$ on topological vector space $X$ is said to be Hypercyclic if there is a vector $x \in X$ whose orbit under $T, \operatorname{Orb}(T, x)=\left\{x, T^{1} x, T^{2} x, \ldots\right\}$ is dense in $X$. In this case $x \in X$ is said to be Hypercyclic vector for $T$ if $\operatorname{Orb}(T, x)$ is dense in $X$.

Definition 1.3. [13] An operator $T: X \rightarrow X$ is multi-hypercyclic (finitely hypercyclic) provided there is a finite subset $\left\{x_{i}, x_{2}, \ldots x_{n}\right\}$ of $X$ such that $\cup_{k=1}^{n} \operatorname{Orb}\left(T, x_{k}\right)$ is dense in $X$.

\section{Main Results}

The extension of Theorem 1.2 from real number to complex number is stated below. The proof of the extended Theorem follows closely the ideas obtained from the real case.

Theorem 2.1. Let $a, b, c, d \in \mathbb{R}$ and $z_{1}=a+i c, z_{2}=b+i d \in \mathbb{C}$. If $a, b>1$ and $\frac{\operatorname{In}\left(z_{1}\right)}{\operatorname{In}\left(z_{2}\right)}$ is irrational then

$$
\left\{\frac{\left(z_{1}\right)^{n}}{\left(z_{2}\right)^{k}}: n, k \in \mathbb{N}\right\} \text { is dense in } \mathbb{R}^{+} .
$$

Proof. Our proof relies on reducing a fraction $\left(\frac{\operatorname{In}\left(z_{1}\right)}{\operatorname{In}\left(z_{2}\right)}\right)$ to a fraction $\left(\frac{\operatorname{In}(a)}{\operatorname{In}(b)}\right)$, and then apply Theorem 1.2. We consider several cases as follows:

Case 2.1. Suppose that $a, b, c, d \in \mathbb{R} \backslash\{0\}$ such that $a, b>1, a=c$ and $b=d$, and $\frac{\operatorname{In}(a+i c)}{\operatorname{In}(b+i d)}$ is irrational, then $\left\{\frac{(a+i c)^{n}}{(b+i d)^{k}}\right\}$ is dense in $\mathbb{R}^{+}$if $n=k \in \mathbb{N}$.

Indeed the one dimensional version of Kronecker's theorem (Theorem 1.1) can be rephrased as follows: If $\theta$ is a positive irrational number, then $\{n \theta-k: n, k \in \mathbb{N}\}$ is dense in $\mathbb{R}$. Now, applying Kronecker's theorem with $\theta=\frac{\ln (a+i c)}{\ln (b+i d)}$ gives that

$$
\left\{n \frac{\ln (a+i c)}{\ln (b+i d)}-k: n=k \in \mathbb{N}\right\} \text { is dense in } \mathbb{R} .
$$

Multiplying by $\ln (b+i d)$ on both sides, we obtain,

$$
\{n \ln (a+i c)-k \ln (b+i d): n=k \in \mathbb{N}\}
$$

is dense in $\mathbb{R}$. By simplifying we get,

$$
\left\{\ln \left(\frac{(a+i c)^{n}}{(b+i d)^{k}}\right): n=k \in \mathbb{N}\right\} \text { is dense in } \mathbb{R} .
$$

Thus, by taking the exponential of the above set, we have that

$$
\left\{\frac{(a+i c)^{n}}{(b+i d)^{k}}: n=k \in \mathbb{N}\right\} \text { is dense in } \mathbb{R}^{+} .
$$

Case 2.2. If $a, b=0$ and $c, d \in\left\{\mathbb{R}^{+} \backslash 0\right\}$ such that $c, d>1$, and $\frac{\ln (a+i c)}{\ln (b+i d)}$ is irrational, then $\left\{\frac{(a+i c)^{n}}{(b+i d)^{k}}: n=k \in \mathbb{N}\right\}$ is dense in $\mathbb{R}^{+}$.

Just as in the case (i) above we have that $\{n \theta-k: n=k \in \mathbb{N}\}$ is dense in $\mathbb{R}$. By applying Kronecker's theorem with $\theta=\frac{\ln (a+i c)}{\ln (b+i d)}$ one has 


$$
\left\{n \frac{\ln (a+i c)}{\ln (b+i d)}-k: n=k \in \mathbb{N}\right\} \text { is dense in } \mathbb{R} \text {. }
$$

Since $a, b=0$, then we have,

$$
\left\{n \frac{\ln (i c)}{\ln (i d)}-k: n=k \in \mathbb{N}\right\} \text { is dense in } \mathbb{R} .
$$

Multiplying by $\ln (i d)$ on both sides, results into,

$$
\{n \ln (i c)-k \ln (i d): n=k \in \mathbb{N}\}
$$

is dense in $\mathbb{R}$. By simplifying, one has,

$$
\left\{\ln \left(\frac{(i c)^{n}}{(i d)^{k}}\right): n=k \in \mathbb{N}\right\} \text { is dense in } \mathbb{R} .
$$

Thus, by taking the exponential of the above set, we see that

$$
\left\{\frac{(i c)^{n}}{(i d)^{k}}: n=k \in \mathbb{N}\right\} \text { is dense in } \mathbb{R}^{+} .
$$

Hence we have

$$
\left\{\frac{c^{n}}{d^{k}}: n=k \in N\right\} \text { is dense in } \mathbb{R}^{+} .
$$

Case 2.3. If $a, b>1, c, d=0$, and $\frac{\operatorname{In}(a+i c)}{\operatorname{In}(b+i d)}$ is irrational or relatively prime integer, then $\left\{\frac{(a+i c)^{n}}{(b+i d)^{k}}: n, k \in \mathbb{N}\right\}$ is dense in $\mathbb{R}^{+}$.

Applying Kronecker's theorem with $\theta=\frac{\ln (a+i c)}{\ln (b+i d)}$ gives that,

$$
\left\{n \frac{\ln (a+i c)}{\ln (b+i d)}-k: n, k \in \mathbb{N}\right\} \text { is dense in } \mathbb{R} \text {. }
$$

Multiplying by $\ln (b+i d)$ on both sides, we have that,

$$
\{n \ln (a+i c)-k \ln (b+i d): n, k \in \mathbb{N}\}
$$

is dense in $\mathbb{R}$. By simplifying, one has,

$$
\left\{\ln \left(\frac{(a+i c)^{n}}{(b+i d)^{k}}\right): n, k \in \mathbb{N}\right\} \text { is dense in } \mathbb{R}
$$

since $c, d=0$, then

$$
\left\{\ln \left(\frac{(a)^{n}}{(b)^{k}}\right): n, k \in \mathbb{N}\right\} \text { is dense in } \mathbb{R} .
$$

Thus, by taking the exponential of the above set, we see that

$$
\left\{\frac{a^{n}}{b^{k}}: n, k \in \mathbb{N}\right\} \text { is dense in } \mathbb{R}^{+} .
$$

Case 2.4. If $a, b \in \mathbb{C} \backslash\{0\}$ and $\left(\frac{\ln (a)^{n}}{\ln (b)^{k}}\right)$ is irrational then $\left\{\frac{a^{n}}{b^{k}}: n, k \in \mathbb{N}\right\}$ is dense in $\mathbb{R}^{+}$.

Let $a=e^{q \pi i}$ and $b=e^{p \pi i}$ where $p, q \in \mathbb{R}$ and $p \neq q$.

Applying Kronecker's theorem with $\theta=\frac{\ln (a)}{\ln (b)}$ gives that,

$$
\begin{gathered}
\left\{n \frac{\ln (a)}{\ln (b)}-k: n, k \in \mathbb{N}\right\} \text { is dense in } \mathbb{R} . \\
\text { that is }\left\{n \frac{\ln \left(e^{q \pi i}\right)}{\ln \left(e^{p \pi i}\right)}-k: n, k \in \mathbb{N}\right\} \text {. By simplifying, one has, } \\
\left\{n \frac{q \pi i}{p \pi i}-k: n, k \in \mathbb{N}\right\} \text { is dense in } \mathbb{R} .
\end{gathered}
$$

then

$$
\left\{n \frac{q}{p}-k: n, k \in \mathbb{N}\right\} \text { is dense in } \mathbb{R} .
$$

Multiplying by $\ln (p)$ on both sides, we have that

$$
\{n q-p k: n, k \in \mathbb{N}\} \text { is dense in } \mathbb{R}^{+} \text {or } \mathbb{R}^{-} .
$$


Note: Also if $p$ or $q$ is positive or negative the answer can either be dense in $\mathbb{R}^{+}$or dense in $\mathbb{R}^{-}$but will still be somewhere dense.

Remark 2.1. The above cases are not exhaustive in the sense that the two cases below remain open.

The first open case is due to the fact that our requirement to reduce $\frac{\ln \left(z_{1}\right)}{\ln \left(z_{2}\right)}$ to $\frac{\ln (a)}{\ln (b)}$ fails and hence Theorem 1.2 can not be used.

Case 2.5. Let $a, b, c, d \in \mathbb{R}$ such that $a, b>1, c, d \neq 0, a \neq c, b \neq d$ then $\frac{\ln (a+i c)}{\ln (b+i d)}$ is a complex number, Hence we can't apply Theorem 1.2.

The second open case obeys the reduction of $\frac{\ln \left(z_{1}\right)}{\ln \left(z_{2}\right)}$ to $\frac{\ln (a)}{\ln (b)}$ but $\frac{\left(z_{1}\right)^{n}}{\left(z_{2}\right)^{k}} \in \mathbb{C}$ if $\left.n \neq k \in \mathbb{N}\right\}$.

Case 2.6. If $a, b=0$ and $c, d \in\left\{\mathbb{R}^{+} \backslash 0\right\}$ such that $c, d>1$, and $\frac{\ln (a+i c)}{\ln (b+i d)}$ is irrational, but if $n \neq k, \frac{\left(z_{1}\right)^{n}}{\left(z_{2}\right)^{k}} \in \mathbb{C}$, therefore it can't be dense in $\mathbb{R}^{+}$.

The following result due to Feldman [11] is useful in constructing both examples:

Corollary 2.1. ([11]) If $a, b>1$ are relatively prime integers, then $\left\{\frac{a^{n}}{b^{k}}: n, k \in N\right\}$ is dense in $\mathbb{R}^{+}$.

We state and give the proof of the following proposition below to show the existence of a somewhere dense orbit in complex plane.

Proposition 2.1. Set $M_{n}(\lambda)=\frac{I+\lambda+\lambda^{2}+\ldots+\lambda^{n-1}}{n}$. If $\lambda$ and $z_{0}$ are complex numbers, then the set $\left\{M_{n}(\lambda) z_{0}\right\}_{n \geq 1}$ of is not dense in $\mathbb{C}$.

Proof. Given a complex number $\lambda$ and let $M_{n} \lambda$ denoted the arithemetic mean of the power of $\lambda \in \mathbb{C}$ and observe that $|\lambda|>1$, then $M_{n}(\lambda)$ diverge to $\infty$.

The uniform ergodic theory deals with the asymptotic behavior of the arithmetic means

$$
M_{n}(\lambda)=\frac{I+\lambda+\lambda^{2}+\ldots+\lambda^{n-1}}{n}
$$

in the operator norm (uniform) topology, as $n \longrightarrow \infty$.

We will show that $\left\{\overline{M_{n}(\lambda) z_{0}: z_{0}, \lambda \in \mathbb{C}}\right\} \neq \mathbb{C}$

$$
\begin{gathered}
M_{1} \lambda=1, \\
M_{2} \lambda=\frac{1+\lambda}{2}, \\
M_{3} \lambda=\frac{1+\lambda+\lambda^{2}}{3}, \\
M_{4} \lambda=\frac{1+\lambda+\lambda^{2}+\lambda^{3}}{4} \ldots . M_{n} \lambda=\frac{1+\lambda+\lambda^{2}+\lambda^{3}+\ldots+\lambda^{n-1}}{n} .
\end{gathered}
$$

and

$$
M_{n} \lambda z_{0}=\frac{1+\lambda+\lambda^{2}+\lambda^{3}+\ldots+\lambda^{n-1}}{n} z_{0} .
$$

We consider, when $|\lambda|>1$ and $|\lambda| \leq 1$,

and we know that $\left|z_{1}+z_{2}\right| \leq\left|z_{1}\right|+\left|z_{2}\right| \Longrightarrow\left|\sum_{i=1}^{n} z_{i}\right| \leq \sum_{i=1}^{n}\left|z_{i}\right|$.

So, $\left|M_{n} \lambda z_{0}\right|=\left|\frac{1+\lambda+\lambda^{2}+\lambda^{3}+\ldots+\lambda^{n-1}}{n} z_{0}\right|$

$$
\begin{gathered}
=\frac{1}{n}\left|1+\lambda+\lambda^{2}+\lambda^{3}+\ldots+\lambda^{n-1}\right|\left|z_{0}\right| \leq \frac{1}{n}\left|z_{0}\right|\left[1+|\lambda|+\left|\lambda^{2}\right|+\left|\lambda^{3}\right|+\ldots+\left|\lambda^{n-1}\right|\right] \\
=\frac{1}{n}\left|z_{0}\right|\left[1+|\lambda|+|\lambda|^{2}+|\lambda|^{3}+\ldots+|\lambda|^{n-1}\right]
\end{gathered}
$$

As $|\lambda| \leq 1$, we have 


$$
\begin{gathered}
\left|M_{n} \lambda z_{0}\right| \leq \frac{1}{n}\left|z_{0}\right|\left[1+|\lambda|+|\lambda|^{2}+|\lambda|^{3}+\ldots+|\lambda|^{n-1}\right] \\
\left|M_{n} \lambda z_{0}\right| \leq \frac{1}{n}\left|z_{0}\right|\left(1+(1)+(1)^{2}+(1)^{3}+\ldots+(1)^{n-1}\right) \\
\leq \frac{1}{n}\left|z_{0}\right|(1+(n-1)) \\
=\frac{1}{n}\left|z_{0}\right|(n)=\left|z_{0}\right| .
\end{gathered}
$$

Therefore, $\left|M_{n} \lambda z_{0}\right| \leq\left|z_{0}\right|$. This means that $M_{n} \lambda z_{0}$ is contained in disc of radius $z_{0}$. If $|\lambda|>1$

$$
\begin{gathered}
\text { as } n \longrightarrow \infty \text {, then } \\
M_{n} \lambda z_{0} \longrightarrow z_{0} \text { for }|\lambda| \leq 1 \text { and }\left|z_{0}\right|>1 \text { for } \lambda>1 .
\end{gathered}
$$

So, $M_{n} \lambda z_{0} \longrightarrow z_{0} \in \mathbb{C}$.

Hence, $\quad\left\{\overline{M_{n} \lambda z_{0}}\right\} \neq \mathbb{C}$ and $\left\{\overline{M_{n} \lambda z_{0}}\right\} \neq \emptyset$.

Therefore, $\left\{M_{n} \lambda z_{0}\right\}_{n \geq 1}$ is contained in the closed unit disk for any positive integer $n$ and $\left\{M_{n} \lambda z_{0}\right\}_{n \geq 1}$ is somewhere dense in complex space but not dense in $\mathbb{C}$.

The following Corollary due to Ansari [3] can be usedful on our results.

Corollary 2.2. (Ansari's Theorem)([3]) If $T$ is hypercyclic, then for every positive integer $n$, the operator $T^{n}$ is also hypercyclic; moreover $T$ and $T^{n}$ share the same collection of hypercyclic vectors.

We give the different proof of Corollary 2.2 to check whether the set of complex numbers has somewhere dense orbits.

Corollary 2.3. If $T$ is hypercyclic, then for every positive integer $n$, the operator $T^{n}$ is also hypercyclic; moreover $T$ and $T^{n}$ share the same collection of hypercyclic vectors.

Proof. We shall prove the $n=3$ on complex case. Suppose that if $T: X \longrightarrow X$ is hypercyclic and $n$ is positive integer that means $\operatorname{Ort}(T, x)$ is dense.

Let $x \in X$.

Now, if $n=1$

$$
\operatorname{Ort}(T, x)=\left\{T^{3 n} x\right\} \cup\left\{T^{3 n}(T x)\right\}=\operatorname{Orb}\left(T^{3} x\right) \cup \operatorname{Orb}\left(T^{3}, T x\right) .
$$

By taking closure, we have

$$
X=\overline{\operatorname{Orb}\left(T^{3} x\right)} \cup \overline{\operatorname{Orb}\left(T^{3}, T x\right)} .
$$

Also by appling the intersection of interior of closure, we get

$$
\operatorname{int}\left(\overline{\operatorname{Orb}\left(T^{3} x\right)}\right) \cap \operatorname{int}\left(\overline{\operatorname{Orb}\left(T^{3}, T x\right)}\right) \neq \emptyset .
$$

If $x$ is hypercyclic vector for $T$ then either $\operatorname{Orb}\left(T^{3} x\right)$ or $\operatorname{Orb}\left(T^{3}, T x\right)$ is somewhere dense that are not dense if and only if $\operatorname{Ort}(T, x)$ is dense in $X$ by above condition. Hence, we have

$$
\operatorname{Orb}\left(T^{3} x\right) \cup \operatorname{Orb}\left(T^{3}, T x\right) \text { is dense in } \mathbb{R} .
$$

Thus $T^{3}$ is hypercyclic, if $x$ is hypercyclic vector for $T^{3}$. But also a weaker property than hypercyclicity is the property of admitting a somewhere dense orbit by using Theorem 3.6 (Feldman 2008) and it is obviously, if $x \in H C(T)$, then $\operatorname{Orb}(x, T)$ is somewhere dense.

We end this paper by giving two examples showing that somewhere dense orbits are not necessarity dense. The first example was given by Feldman [11] in real Hilbert space, and the second examples is ours which is an extension of the first example to the complex Hilbert space. 


\section{Example 2.1.}

(i) Let $I$ be the identity operator on the complex Hilbert space $\mathbb{C}$ and let $v_{1}=i$ and $v_{2}=-i$. If we let $T=\left(T_{1}, T_{2}\right)$ where $T_{1}=2 I$ and $T_{2}=\frac{1}{3} I$, then by Corollary 2.1 due to Feldman [11], $\operatorname{cl}(\operatorname{Orb}(T, i))=[0, \infty) i$ and $\operatorname{cl}(\operatorname{Orb}(T,-i))=(-\infty, 0] i$. Hence $T$ has somewhere dense orbits that are not dense. Furthermore, $\operatorname{Orb}(T, i) \cup \operatorname{Orb}(T,-i)$ is dense in $\mathbb{C}$, but $T$ is not hypercyclic. Thus $T$ is multi-hypercyclic, but not hypercyclic.

(ii) Let

$$
\begin{aligned}
& T_{1}=\left[\begin{array}{ll}
2 i & \\
& i
\end{array}\right], T_{2}=\left[\begin{array}{ll}
\frac{1}{3} i & \\
& i
\end{array}\right], T_{3}=\left[\begin{array}{ll}
i & \\
& 2 i
\end{array}\right], T_{4}=\left[\begin{array}{ll}
i & \\
& \frac{1}{3} i
\end{array}\right] \\
& T=\left(T_{1}, T_{2}, T_{3}, T_{4}\right) \text {, and } v_{i}, j_{j}=\left[\begin{array}{l}
(-1)^{i} \\
(-1)^{j}
\end{array}\right] \text { where } i, j \in\{0,1\} \text {. Then the vectors } v_{0,0}, v_{1,0}, v_{1,1}, v_{0,1} \text { lie in }
\end{aligned}
$$
the first, second, third, and fourth quadrants of $\mathbb{R}^{2}$ respectively. Furthermore, Orb $\left(T, v_{i, j}\right)$ is dense in the quadrant that contains $v_{i, j}$. Thus we see that $T$ has somewhere dense orbits that are not dense in $\mathbb{C}^{2}$ and that $T$ is multi-hypercyclic but not hypercyclic. In fact, $T$ is 4-hypercyclic, but not 3-hypercyclic (since each of the 4 quadrants are invariant sets for $T$ with disjoint interiors).

\section{References}

[1] Al-shami T. M., Somewhere dense set and st-space, J. Appl. Math, 49(2): 101-111, 2017.

[2] Ansari S. I., Hypercyclic and cyclic vectors, J. Funct. Anal, 128(2): 374-383, 1995.

[3] Ansari S. I., Existence of hypercyclic operator on topological vector spaces, J. Funct. Anal, 148(2): 384-390, 1997.

[4] Ayadi A., Hypercyclic abelian semigroup of matrices on $\mathbb{C}^{n}$ and $\mathbb{R}^{n}$ and k-transitivity, J. Appl. General Topology, 12(1): 35-39, 2011.

[5] Bayart F., É. Matheron, Dynamics of linear operators, Cambriage university press, vol. 179: 2009.

[6] Bayart F., Costakis G., Hypercyclic operators and rotated orbits with polynomial phases, J. London Math., 89(3): 663-679, 2014.

[7] Bourdon P. S., Feldman N. S., Somewhere dense orbits are everywhere dense, Indiniana University Math., 52(3): 811-819, 2003.

[8] Boyd D. W., Kronecker's theorem and Lehmer's problem for polynomials in several variables, Journal of Number Theory, 13(1): 116-121, 1981.

[9] Costakis G., Peris A., Hypercyclic semigroups and somewhere dense orbits, Comptes Rendus Mathematique, 335(11): 895898, 2002.

[10] Costakis G., Hadjiloucas D., Somewhere dense Cesáro orbits and rotations of Cesáro hypercyclic operators, Studia Mathematica, Vol. 175: 249-269, 2006.

[11] Feldman N. S., Hypercyclic tuples of operators and somewhere dense orbits, J. Math Anal. Appl., 346(1): 82-98, 2008.

[12] Leon-Saavedra F., Operators with hypercyclic Cesáro means, Studia Mathematica, 152(3): 201-215, 2002.

[13] Peris A., Multi-hypercyclic operators are hypercyclic, Mathematische Zeitschrift, 236(4): 779-786, 2001.

[14] Rolewicz S., On orbits of elements, Studia Mathematica, 32(1): 17-22, 1969. 